\title{
Modulation of SRSF2 expression reverses the exhaustion of TILs via the epigenetic regulation of immune checkpoint molecules
}

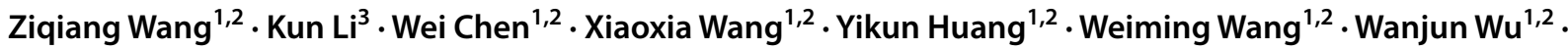 \\ Zhiming $\mathrm{Cai}^{1,2} \cdot$ Weiren Huang ${ }^{1,2}$ (1)
}

Received: 14 March 2019 / Revised: 15 October 2019 / Accepted: 30 October 2019 / Published online: 14 December 2019

(c) The Author(s) 2019

\begin{abstract}
The elevated expression of immune checkpoints by the tumor microenvironment is associated with poor prognosis in several cancers due to the exhaustion of tumor-infiltrating lymphocytes (TILs), and the effective suppression of the expression of these genes is key to reversing the exhaustion of TILs. Herein, we determined that serine/arginine-rich splicing factor 2 (SRSF2) is a target for blocking the tumor microenvironment-associated immunosuppressive effects. We found that the expression of SRSF2 was increased in exhausted T cells and that SRSF2 was involved in multiple immune checkpoint molecules mediating TILs' exhaustion. Furthermore, SRSF2 was revealed to regulate the transcription of these immune checkpoint genes by associating with an acyl-transferases P300/CBP complex and altering the H3K27Ac level near these genes, thereafter influencing the recruitment of signal transducer and activator of transcription 3 (STAT3) to these gene promoters. Collectively, our data indicated that SRSF2 functions as a modulator of the anti-tumor response of T cells and may be a therapeutic target for reversing the exhaustion of TILs.
\end{abstract}

Keywords T-cell exhaustion $\cdot$ Immune checkpoint $\cdot \mathrm{SRSF} 2 \cdot$ Epigenetic regulation $\cdot$ Histone modification

Electronic supplementary material The online version of this article (https://doi.org/10.1007/s00018-019-03362-4) contains supplementary material, which is available to authorized users.

Ziqiang Wang, Kun Li, and Wei Chen have contributed equally to this work.

Zhiming Cai

caizhiming2000@163.com

$\triangle$ Weiren Huang

pony8980@163.com

1 Department of Urology, Shenzhen Second People's Hospital, The First Affiliated Hospital of Shenzhen University, International Cancer Center, Shenzhen University School of Medicine, Shenzhen 518039, China

2 Guangdong Key Laboratory of Systems Biology and Synthetic Biology for Urogenital Tumors, Shenzhen 518035, China

3 Department of Nuclear Medicine, Shandong Provincial Qianfoshan Hospital, The First Hospital Affiliated with Shandong First Medical University, Jinan 250014, China

\section{Introduction}

Renal cell carcinoma (RCC) is the most common type of kidney cancer and accounts for $90-95 \%$ of all kidney cancer diagnosis and 3\% of adult malignancies [1]. While there are multiple conventional treatments available for RCC including surgery, chemotherapy, and radiotherapy, there is a subset of RCC patients (30\%) that exhibit metastases and are unresponsive to chemotherapy and radiotherapy [2]. It is known that both innate and adaptive immune cells with RCC specificity develop naturally in most patients, and lymphocytes are recruited to the tumor to secrete cytokines $[3,4]$. However, higher numbers of T cells in RCC tissue correlates with a poorer prognosis as there are limitations on immune cells and the capacity with which they exert their effector functions $[5,6]$. This suggests that for some unknown reasons, RCC T cells are unable to produce an immune response against the tumor cells and control tumor growth. Recent studies have suggested that the immunosuppressive effects of the tumor microenvironment may be the cause of this T-cell failure in RCC tissue.

In the tumor microenvironment, there is an ongoing balance between the cytotoxic T lymphocytes (CTLs) that work 
to eliminate tumor cells through the secretion of cytokines including IFN- $\gamma$ and IL-2, and the tumor cells themselves that can negatively regulate CTLs through the association with other immunosuppressive cells like myeloid-derived suppressor cells (MDSCs), tumor-associated macrophages (TAMs), and regulatory T cells (Tregs) [7]. These immunosuppressive cells can secrete cytokines like IL-6, IL-10, TGF- $\beta$, and VEGF $[8,9]$ that can have a direct inhibitory effect on the activation of CTLs by stimulating expression of immune checkpoint molecules that represent cell exhaustion on $\mathrm{T}$ cells [10]. There are many immune checkpoint molecules including programmed cell death 1 (PD-1), B- and T-lymphocyte attenuator (BTLA), T-cell immunoglobulin and mucin-domain containing-3 (TIM-3), lymphocyte activation gene 3 (LAG-3), cytotoxic T-lymphocyte antigen-4 (CTLA-4), T-cell immunoreceptor with immunoglobulin, and immunoreceptor tyrosine-based inhibitory motif domains (TIGIT) and CD160. These immune checkpoint molecules contribute to CTLs dysfunction in cytokine secretion, proliferation, tumor cell cytotoxicity, and effective memory cell generation. Therefore, understanding how to directly downregulate different immune checkpoint molecules expression represents a key issue toward reversing CTLs' dysfunction.

There is a growing body of evidence confirming the involvement of epigenetic regulation in T-cell dysfunction. Research from the Dana Farber Cancer Institute and Harvard-MIT Broad Institute found that BAF 180, a protein localized to the chromatin remodeling complex SWI/SNF, regulates the expression of multiple immune checkpoint molecules, thereby mediating T-cell function and T-cellmediated anti-tumor immune responses $[11,12]$. In addition, histone deacetylase inhibitors also regulate the antitumor immune response of tumor-infiltrating CD4 + T cells [13]. These studies reveal that epigenetic mechanisms play an important role in regulating tumor-infiltrating lymphocytes (TILs) function. In this study, we look for epigenetic mechanisms of immune checkpoint molecules that mediate T-cell processes and find that serine/arginine-rich splicing factor 2 (SRSF2) is required for regulating T-cell function.

SRSF2 is an important component of nuclear structure speckle, with many reported functions including genomic stability maintenance, pre-mRNA splicing, and nuclear output regulation and aiding in mRNA translation [14-18]. Through the regulation of cancer-associated splice variants, SRSF2 have been implicated in many types of cancer, including lung carcinoma [19], hepatocellular carcinoma [20], and RCC [21]. Additionally, SRSF2 has been associated with a variety of immune disorders including systemic lupus erythematosus (SLE) [22], leukemia [23], and human immunodeficiency virus infection [24]. Furthermore, SRSF2 is known to regulate gene expression of many T-cell molecules, such as CD45 [25], CD44 [26], and costimulatory signal molecule B7-H3 [27]. Previously, we found that SRSF2 directly binds to genomic DNA to regulate the gene's transcriptional activity [28], suggesting that SRSF2 may regulate T-cell functions through a similar epigenetic regulation mechanism.

In this study, we found that tumor cells isolated from RCC tissue did not trigger the immune response of TILs and downregulation of SRSF2 in these TILs could significantly improve their immune response against tumor cells. Upon further analysis, we found that RCC TILs overexpressed multiple immune checkpoint molecules, and that inhibition of SRSF2 in TILs led to the downregulation of these immune checkpoint molecules. We found that SRSF2 regulates the transcriptional activities of these genes by altering the nearby $\mathrm{H} 3 \mathrm{~K} 27$ acetylation via the association with acetyl-transferases P300/CBP complex and signal transducer and activator of transcription 3 (STAT3). All these data suggest that SRSF2 may be a therapeutic target to block tumorassociated suppressive pathways.

\section{Results}

\section{SRSF2 modulates the immune response against autologous tumor cells in TILs}

Studies have reported that TILs exhibited cell exhaustion in most solid tumors [29-31]. To determine the status of TILs in RCC, we isolated TILs and tumor cells from primary RCC specimens, co-cultured these cells, and examined IFN- $\gamma$ production using an ELISA assay to investigate the direct immune responses against autologous tumor cells in TILs. The results demonstrated that only tumor cell lines isolated from sample 10 could stimulate the production of IFN- $\gamma$ in TILs (Fig. 1a). However, the downregulation of SRSF2 by SRSF2 siRNA (siSRSF2) (Fig. S1) in these TILs could significantly improve their immune response against tumor cell lines in sample 3, 4, 7, 10, 11, 12, and 13 (Fig. 1b). Moreover, we conducted flow cytometry with antibodies against IFN- $\gamma$ and CD8 in these positive samples, and found that the frequency of IFN $-\gamma+$ and CD $8+$ TILs among the TILs co-cultured with the autologous tumor cell lines was significantly higher than that in the TILs alone, while the expression of SRSF2 in these TILs was downregulated by siSRSF2 (Fig. 1c-i). Taken together, our results show that most TILs isolated from primary RCC specimens could not recognize autologous tumor cells and that SRSF2 functions as an important regulator of the immune response against tumor cells in TILs. 

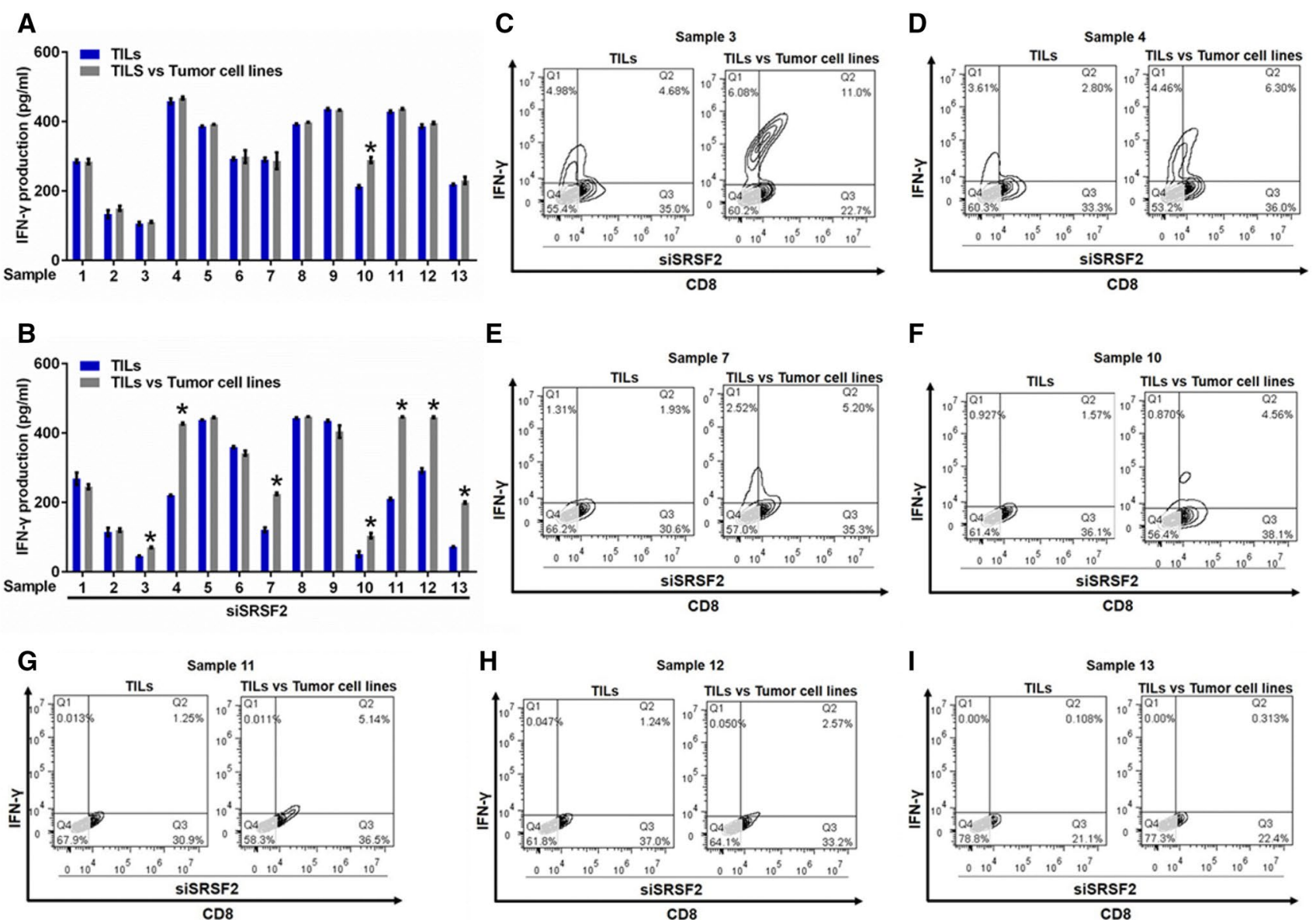

Fig. 1 Inhibition of SRSF2 reversed the dysfunction of TILs. a IFN- $\gamma$ production by TILs was determined in TILs or TILs co-cultured with tumor cell lines using an ELISA assay in three independent experiments. The data are represented as the mean \pm SD. b IFN $-\gamma$ production by TILs was determined in TILs in which the expression of SRSF2 was inhibited by siSRSF2 or these TILs co-cultured with tumor cell lines using an ELISA assay in three independent experi-

\section{SRSF2 regulates the expression of multiple immune checkpoints}

A previous study reported that immune checkpoints on $\mathrm{T}$ cells represent the functional status of these cells [32]. To investigate why the tumor cells could not trigger the immune response in the autologous TILs, we compared the expression level of multiple immune checkpoints between TILs and peripheral blood mononuclear cells (PBMCs) containing many effector T cells. We isolated TILs and PBMCs from tumor tissues and peripheral blood from samples 3, 4, 7, $10,11,12$, and 13. Then, the total RNA was extracted from these cells and real-time PCR was conducted. Our results showed that the expression of PD-L1, BTLA, CTLA4, LAG3, and CD160 was significantly increased in the TILs compared with that in the PBMCs (Fig. 2a-e). For further confirmation, we performed flow cytometry using antibodies ments. The data are represented as the mean \pm SD. $\mathbf{c}-\mathbf{i}$ The frequency of IFN- $\gamma+$ and CD $8+$ TILs was assessed by flow cytometry in TILs in which the expression of SRSF2 was inhibited by siSRSF2 or these TILs co-cultured with tumor cell lines isolated from Sample 3 (c), Sample 4 (d), Sample 7 (e), Sample 10 (f), Sample 11 (g), Sample 12 (h), and Sample 13 (i). ${ }^{*} p<0.01$

against these immune checkpoints, and CD8 in the TILs and PBMCs from sample 13 that exhibited greater alteration in the immune response following the knockdown of SRSF2. The results showed that the frequency of immune checkpoint + and CD8 + TILs was significantly higher among the TILs than that among the PBMCs (Fig. $2 \mathrm{f}-\mathrm{j}$ ).

To uncover the mechanism by which SRSF2 regulates the immune response of autologous TILs, we first used a RCC data set (GSE40435) from the National Center for Biotechnology Information (NCBI) database to analyze SRSF2 expression levels in the RCC tumor tissue and adjacent tumor tissue. As shown in Figure S2, SRSF2 expression was increased in RCC tumor tissue. Then, we examined the expression level of SRSF2 in TILs and PBMCs, and found that the TILs exhibited a higher level than the autologous PBMCs (Fig. 3a). Thereafter, we elucidated the relationship between the expression of SRSF2 and immune checkpoints. 


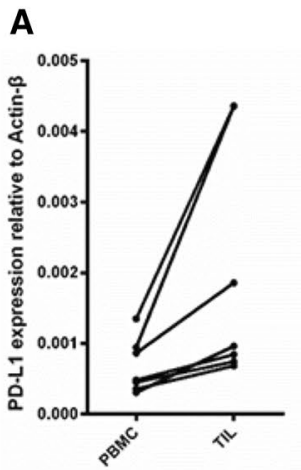

B
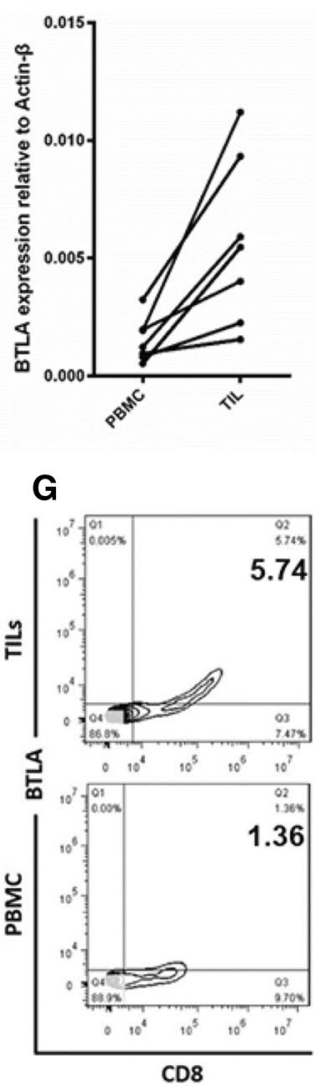

C

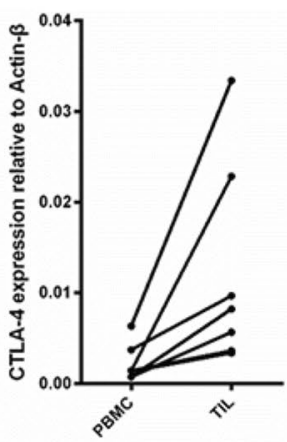

$\mathbf{H}$

D

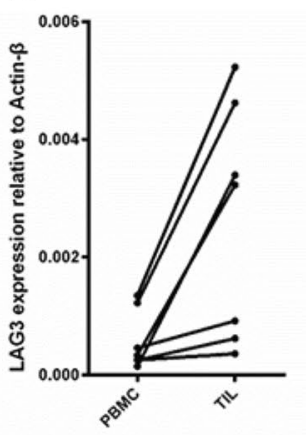

I

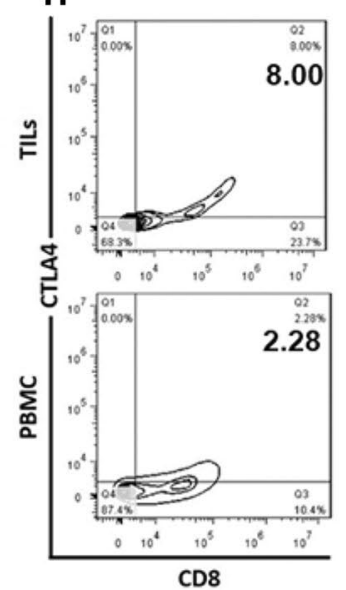

E
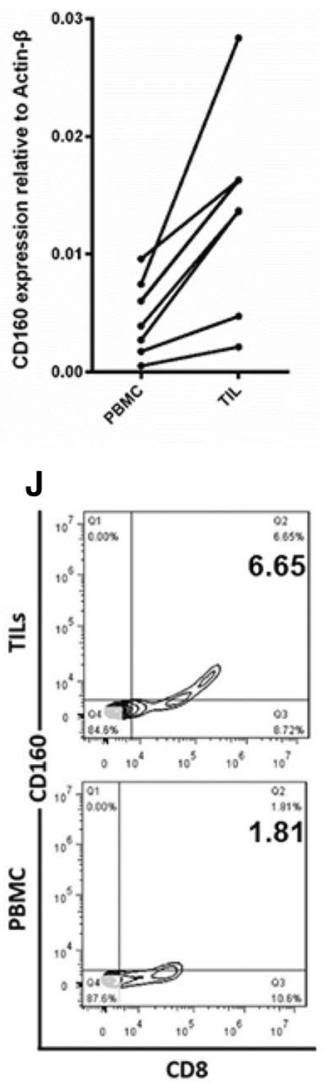

Fig. 2 The elevated expression of immune checkpoint molecules in TILs. The expression level of immune checkpoint molecules was determined in TILs and compared with that in autologous PBMCs by real-time PCR (a-e) and flow cytometry $(\mathbf{f}-\mathbf{j})$

We silenced SRSF2 expression by siSRSF2 and found that the depletion of SRSF2 downregulated the expression of these immune checkpoints (Fig. 3b-f). Taken together, these results demonstrated that TILs isolated from tumor tissues expressed higher levels of multiple immune checkpoints and that SRSF2 regulates the expression of these immune checkpoints.

\section{SRSF2 regulates the transcriptional activities of immune checkpoint genes}

To better understand the mechanism by which SRSF2 regulates these related genes, we verified the regulatory roles of SRSF2 in the expression of these immune checkpoint genes in Jurkate E6, which is a human immortalized T-lymphocyte cell line, and obtained results similar to those obtained in the TILs (Fig. S3). Our previous study illustrated that in addition to participating in pre-mRNA splicing, SRSF2 could function as a transcriptional activator of the expression of related genes by binding the promoters of these genes [28]. Therefore, we conducted a luciferase assay to determine whether SRSF2 directly regulates the transcriptional activities of these immune checkpoints. We generated a luciferase reporter construct containing the promoter of this gene. The results showed that the transcriptional activity of the $P D$ L1, BTLA, CTLA4, LAG3, and CD160 gene promoters in the SRSF2-depleted Jurkate E6 cells was inhibited (Fig. 4a). To determine whether the downregulation of SRSF2 alters histone modifications near the transcriptional start sites (TSS) of these genes, we designed sets of primer pairs that recognize the corresponding TSS regions of these genes (Fig. S4) and performed chromatin immunoprecipitation (ChIP) experiments using antibodies against tri-methylated histone $\mathrm{H} 3$ at lysine 4 (H3K4Me3), acetylated histone $\mathrm{H} 3$ at lysine $27(\mathrm{H} 3 \mathrm{~K} 27 \mathrm{Ac})$, and tri-methylated histone $\mathrm{H} 3$ at lysine 27 (H3K27Me3) in Jurkate E6 cells transfected with SRSF2 siRNAs (siSRSF2) or control siRNAs (siCTRL). In these histone modifications, H3K4Me3 and H3K27Ac at transcription start sites (TSS) serve as markers of actively transcribed genes, while $\mathrm{H} 3 \mathrm{~K} 27 \mathrm{Me} 3$ at TSS is associated with gene repression [33]. The results showed that knocking down SRSF2 decreased the enrichment of H3K27Ac at the promoters of these genes (Fig. 4b-f). Taken together, our results demonstrated that SRSF2 regulates $P D-L 1, B T L A$, 

expression of multiple immune checkpoint genes. a The expression level of SRSF2 was determined in TILs isolated from Sample 3, 4, 7, 10, 11, 12 , and 13 and compared with that in autologous PBMCs by real-time PCR. b-f $36 \mathrm{~h}$ after these TILs were transfected with SRSF2-targeting siRNAs (siSRSF2) or negative control siRNAs (siCTRL), the expression level of PD-L1 (b), BTLA (c), CTLA4 (d), LAG3 (e), and CD160 (f) was determined by
Fig. 3 SRSF2 regulates the real-time PCR
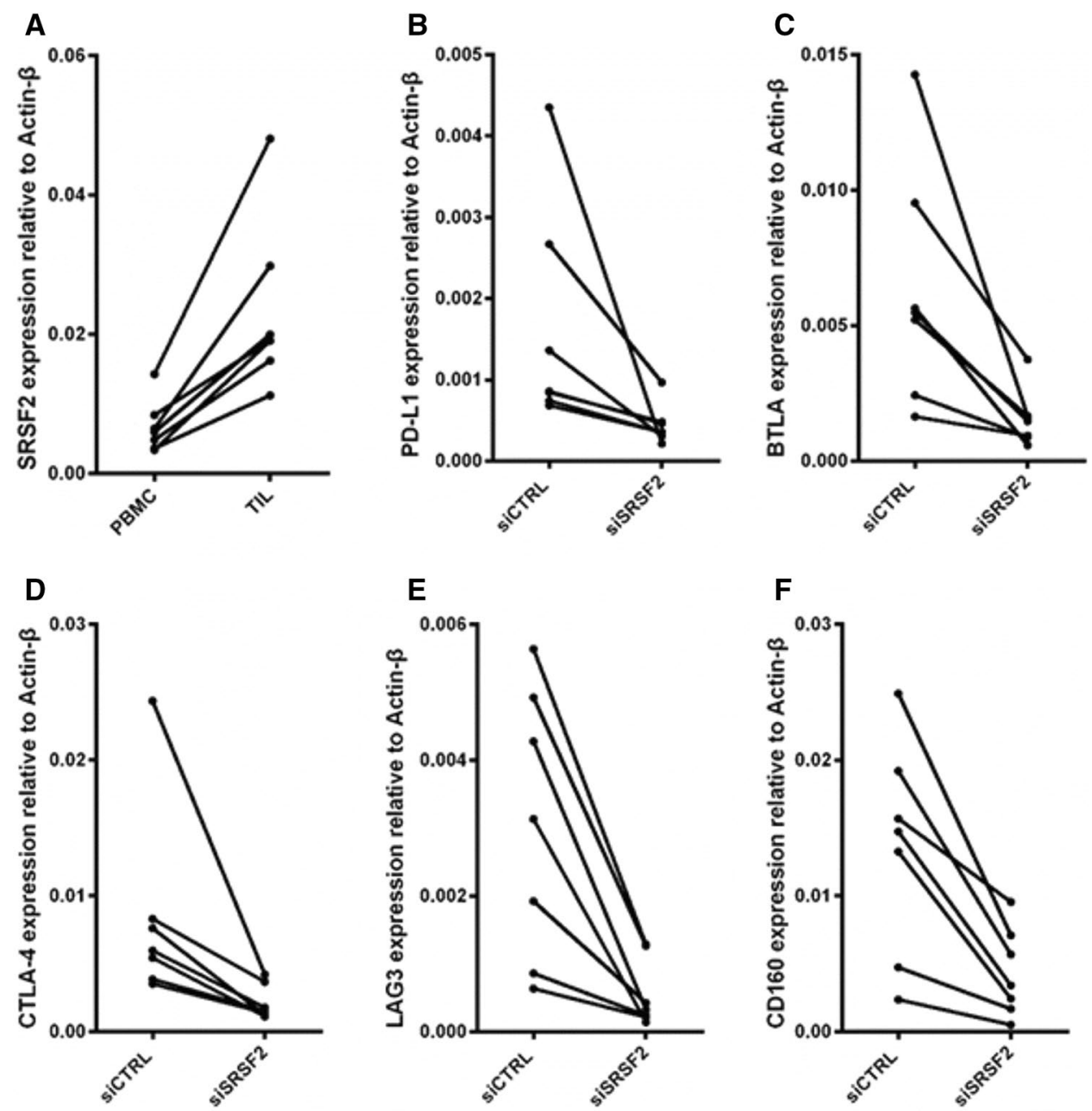

CTLA4, LAG3, and CD160 transcriptional activity by altering the histone modification status of these gene promoters.

\section{SRSF2 is associated with the P300/CBP complex}

To further study the molecular mechanism by which SRSF2 regulates histone acetylation at the promoters of the target genes, we detected and analyzed the effect of SRSF2 on the global levels of H3K27Ac. The results revealed a decrease in H3K27Ac in the SRSF2 knockdown cells (Fig. 5a). Given that histone acetylation requires the acetyl-transferases $\mathrm{P} 300$ / CBP complex and is involved in the epigenetic regulation of gene expression $[34,35]$, in our study, we focused on the relationship between SRSF2 and P300/CBP. Since SRSF2 and P300/CBP are considered transcriptional regulators of great magnitude $[28,34]$ and both regulate histone acetylation, we first conducted immunofluorescence experiments to determine whether SRSF2 is associated with P300/CBP in spatial location. As shown in Fig. 5b, SRSF2 was largely colocalized with P300, and a small amount of SRSF2 was colocalized with CBP. In addition, pixel intensity plots of
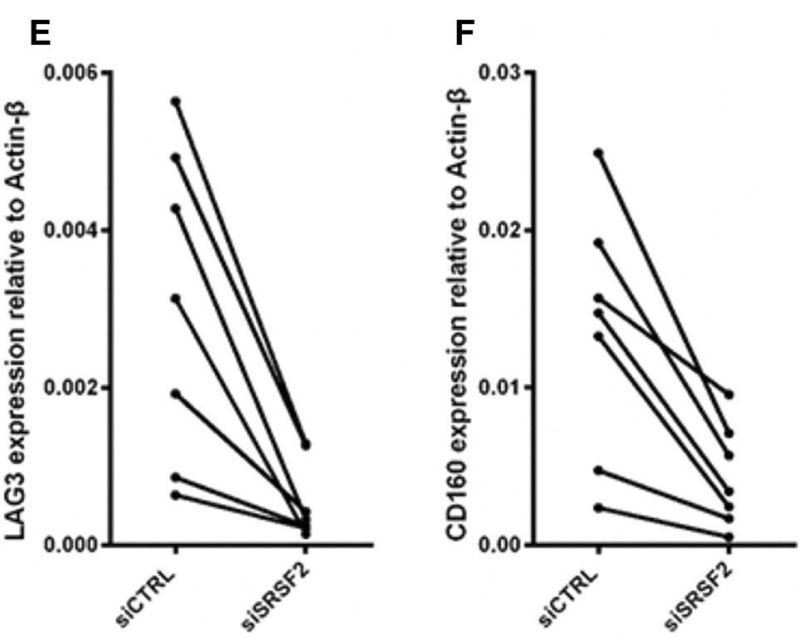

each merged channel were generated by ImageJ software (Fig. 5b, right panels). Additionally, we performed an immunoprecipitation assay to confirm this association. The Jurkate E6 cell lysates were harvested, and an immunoprecipitation assay was performed using antibodies against SRSF2 or IgG, followed by western blotting using an anti-SRSF2 antibody, anti-P300 antibody and anti-CBP antibody. P300 and CBP were indeed specifically pulled down in the cell lysates (Fig. 5c). These data are the first to show that SRSF2 is colocalized with P300/CBP complex to regulate H3K27 acetylation near the TSS of related genes.

\section{SRSF2 is required for the recruitment of STAT3 to H3K27AC}

Given that the H3K27Ac level near the TSS of genes influences the binding ability of STAT3, an important transcriptional factor for gene expression [36], to target genes [37, 38 ], and that STAT3 plays vital roles in inducing tumor immune tolerance [39], we hypothesized that STAT3 is involved in SRSF2-mediated gene expression immune 
A

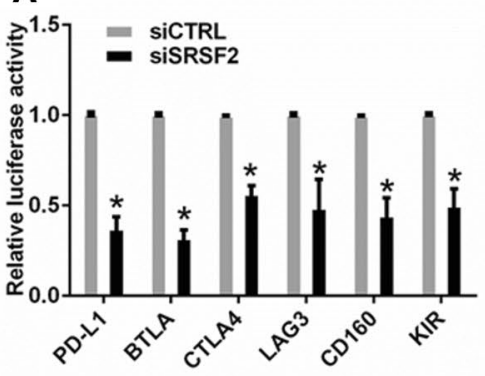

D

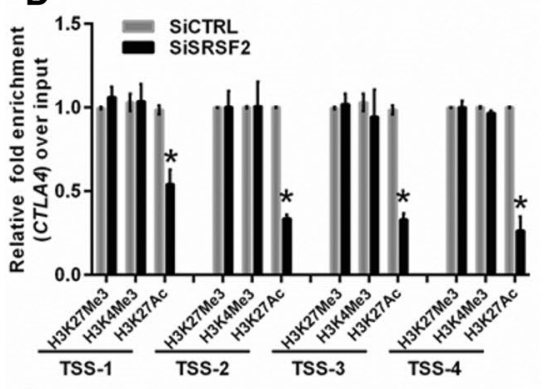

B

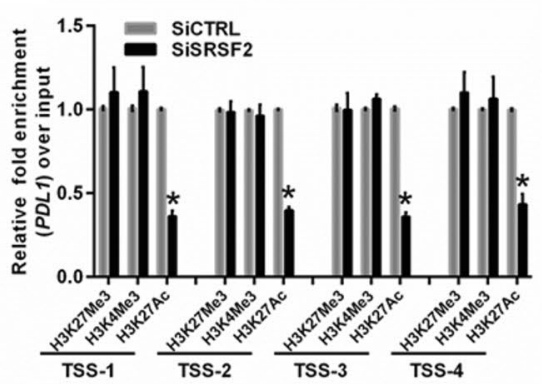

E

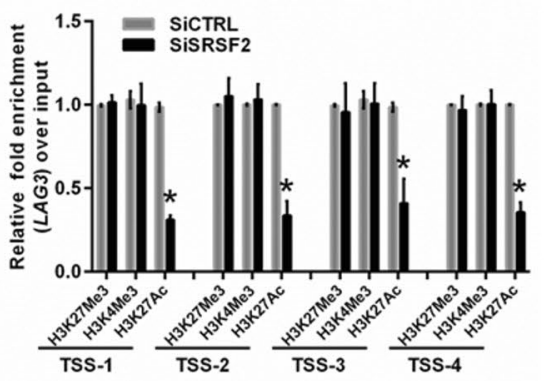

C

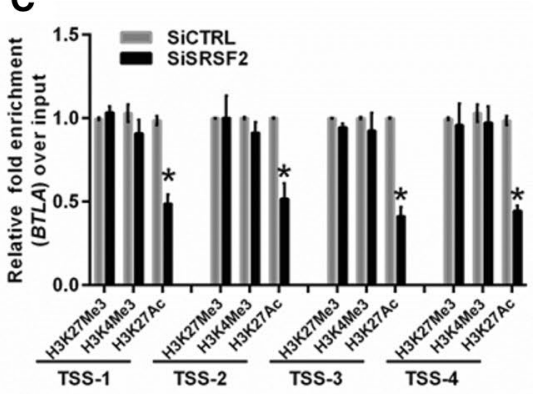

$\mathbf{F}$

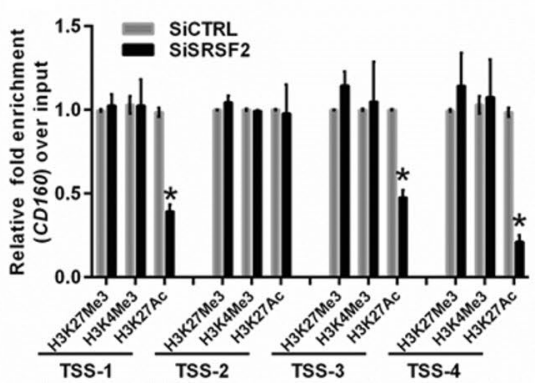

Fig. 4 SRSF2 regulates the transcriptional activities of immune checkpoint genes by regulating histone modification. a After the cotransfection with the SRSF2 siRNAs or negative control siRNAs and the pGL3 enhancer plasmid containing the $P D-L 1$ promoter, $B T L A$ promoter, CTLA-4 promoter, LAG3 promoter or CD160 promoter for $36 \mathrm{~h}$, the relative transcriptional activities of theses promoters were determined with a luciferase assay in three independent experiments. The data are represented as the mean \pm SD. b-f Jurkate

checkpoints. To achieve it, we analyzed STAT3 expression levels in the RCC tumor tissue and adjacent tumor tissue with the RCC data set (GSE40435), and the results demonstrated that STAT3 expression was significantly increased in RCC tumor tissue (Fig. S5A). Additionally, the general consensus STAT3-binding motif [40] was identified at the TSS of these genes (Figure S5B-F), and silencing STAT3 expression with specific STAT3-targeting siRNAs resulted in reduced levels of the expression of these genes (Fig. S5G). Furthermore, the immunofluorescence and immunoprecipitation results showed that STAT3 Y705p, which is the active form of STAT3, is largely colocalized with H3K27Ac and that the inhibition of endogenous SRSF2 expression could disrupt this association between STAT3 Y705p and H3K27Ac (Fig. 6a, b), suggesting that SRSF2 plays a scaffolding role in the interaction between STAT3 Y705p and H3K27Ac. To clarify the mechanism of this association, we performed immunofluorescence with antibodies against SRSF2, H3K27Ac, and STAT3 Y705p, and immunoprecipitation with antibodies against SRSF2 and IgG. The results demonstrated that SRSF2 interacted with both STAT3 Y705p and H3K27Ac (Fig. 6c, d). Also, a ChIP assay with antibody against to STAT3 Y705 showed
E6 cells transfected with SRSF2 siRNAs or negative control siRNAs were collected for ChIP assays to analyze the relative fold enrichment of the $P D-L 1$ promoter (b), BTLA promoter (c), CTLA-4 promoter (d), LAG3 promoter (e), or CD160 promoter (f) by an anti-H3K4Me3 antibody, anti-H3K27Me3 antibody or anti-H3K27Ac antibody. The data points represent mean values determined from three independent experiments. The data are presented as the mean \pm SD. $* p<0.01$

that downregulation of SRSF2 resulted in a decrease in the enrichment of STAT3 Y705p bound to the immune checkpoints genes' promoters (Fig. S6). These results suggested that SRSF2 is required for the recruitment of STAT3 to these immune checkpoint genes.

\section{Discussion}

Since 2013, cancer has become the first cause of death among the urban and rural residents of China. However, the existing treatment methods, such as surgery, radiotherapy, and chemotherapy, cannot effectively cure cancer. Researchers have found that radiotherapy and chemotherapy can cause apoptosis and necrosis in cancer cells. However, these treatments also cause senescence. These senescent cells can promote cancer stemness by activating the Wnt signaling pathway, which can eventually lead to cancer relapse and metastasis [41, 42]. In fact, many tumor-bearing patients naturally develop a complex immune response against tumor activity. However, the effector immune cells in the tumor microenvironment are suppressed by tumor cells and other 
Fig. 5 SRSF2 associates with the P300/CBP complex. a Protein levels of H3K27Ac, Histone $\mathrm{H} 3$ and $\beta$-actin in Jurkate E6 cells transfected with SRSF2 siRNAs or negative control siRNAs were measured by western blotting. b Jurkate E6 cells were cultured in poly-D-lysine,coated plates, fixed, incubated with an anti-SRSF2 antibody (red), anti-P300 antibody (green), or anti-CBP antibody (green), and subjected to a confocal analysis. The intensity plots of the red and green channels were analyzed with ImageJ software. Scale bars, $10 \mu \mathrm{m}$. c Jurkate E6 cells lysates were collected and immuno-precipitated with an anti-SRSF2 antibody or anti-IgG antibody, followed by a western blot analysis of SRSF2, P300, and CBP

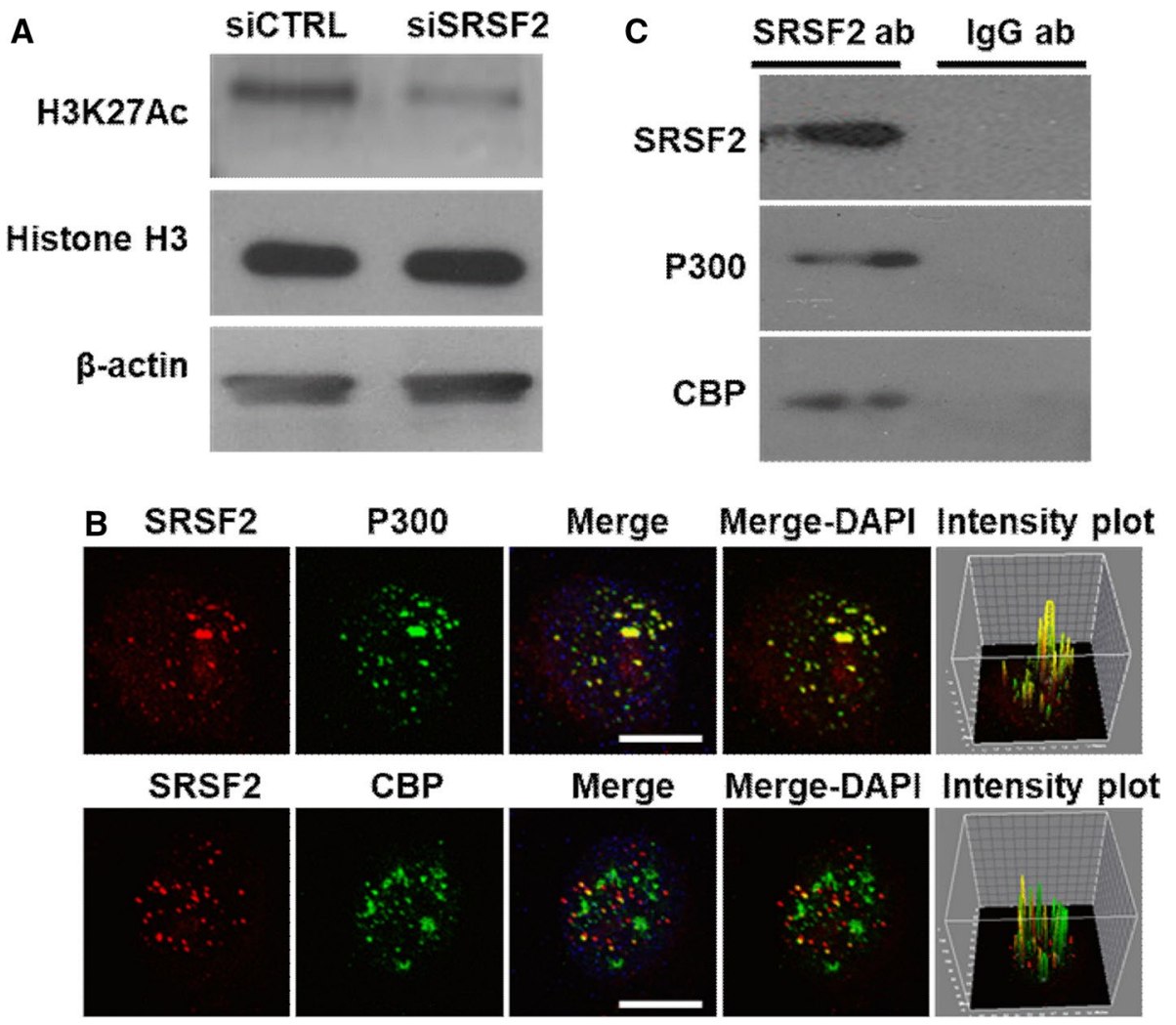

immunosuppressive cells, such as MDSCs, TAMs, and Tregs.

Growing evidence suggests that developing techniques that inhibit the effects of the tumor microenvironment on immune cells could be helpful in enhancing the anti-tumor efficacy of tumor immunotherapy [43]. In recent years, targeted immune checkpoint molecules have been considered an effective therapeutic strategy for eliminating the tumor microenvironment impacts [44]. In the current study, we found that the inhibition of SRSF2 expression in TILs significantly improves its anti-tumor immune response via the epigenetic regulation of multiple immune checkpoint molecules. SRSF2 associates with the P300/CBP complex to regulate its acetyl-transferases activity and H3K27 acetylation. Then, SRSF2 recruits the transcriptional factor STAT3 to H3K27Ac near the TSS of these immune checkpoint genes to regulate gene transcription (Fig. 6e).

In this study, we first found that most TILs in the RCC specimens are in an immunosuppression status with a high expression of multiple immune checkpoint molecules and could not produce an immune response against autologous tumor cells. To be exciting, we found that blocking SRSF2 expression in TILs could reverse its immunosuppression through the downregulation of the expression level of these immune checkpoint genes. To date, many inhibitors targeting immune checkpoint molecules have been approved for the treatment of solid tumors and displayed certain therapeutic efficacy in preclinical and clinical trials [45-50]. However, due to off-target effects, these immunotherapies have led to many immune-related adverse events [51]. Additionally, immune checkpoint inhibitor treatments are considered dangerous for the treatment of patients who have underlying autoimmune diseases or chronic immunosuppression [52]. All these defects are attributed to the off-target effects of these immune checkpoint inhibitors. Therefore, developing a strategy to directly downregulate the expression of this immune checkpoint in vitro before their expansion with reinfusion into the tumor-bearing patient may be a good choice for avoiding these defects. In this study, we found that TILs transfected with SRSF2-targeting siRNAs could reverse the exhaustion of TILs by downregulating the expression of multiple immune checkpoint genes, including PD-L1, BTLA, CTLA4, LAG3, and CD160.

Studies have shown that exhausted T cells in the tumor microenvironment exhibit distinct epigenetic profiles [53, 54] and that immune checkpoint inhibitors, such as antiPD-L1 antibodies, could not alter the epigenetic state to reverse the exhaustion of T cells [54]. In the current study, blocking SRSF2 expression with SRSF2-specific siRNAs could downregulate the level of H3K27Ac near multiple immune checkpoint genes via the disruption of the association between SRSF2 and the acetyl-transferase P300/ CBP complex. Additionally, we found that the depletion of SRSF2 decreases the enrichment of the transcriptional factor 
A
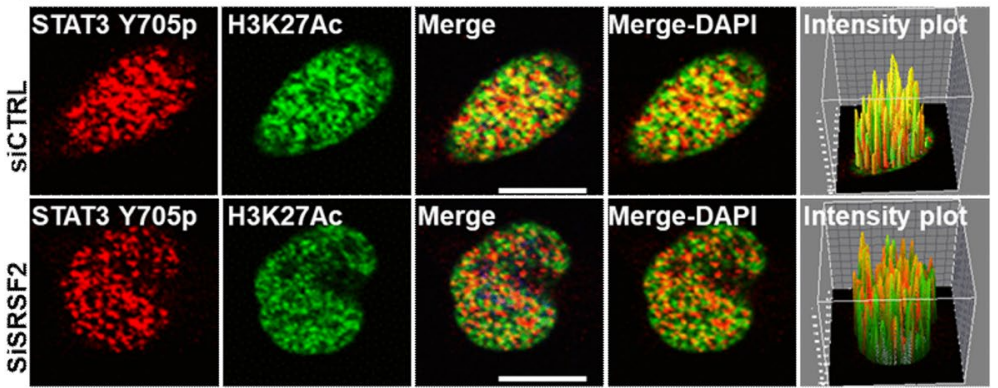

C
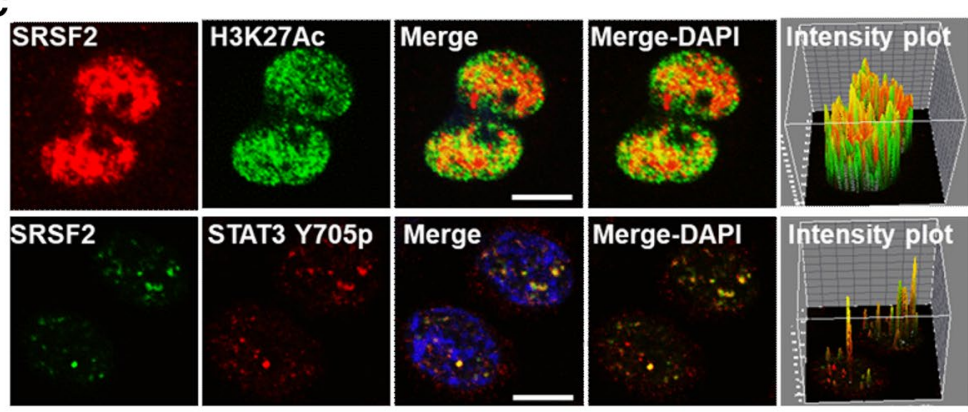

E

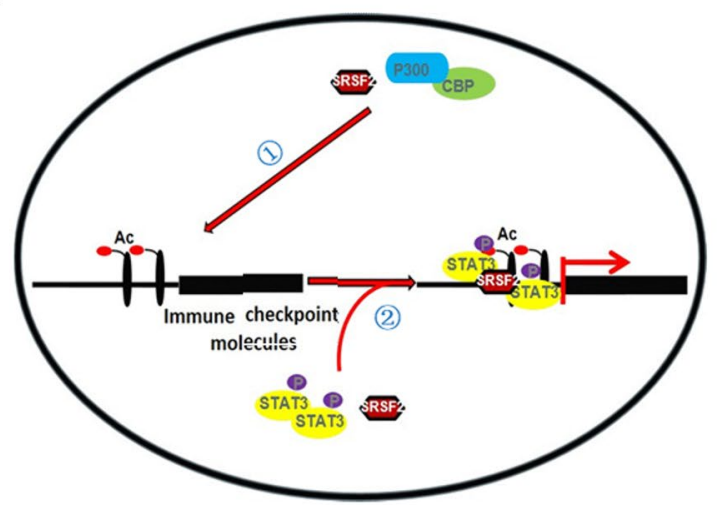

B

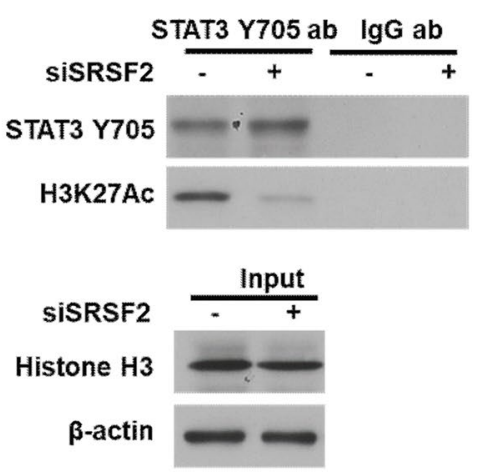

D

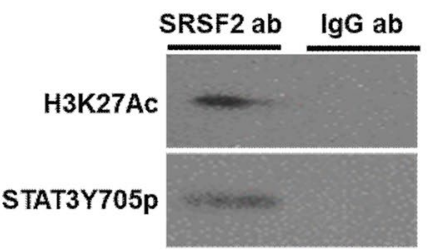

Fig. 6 SRSF2 associates with STAT3 and H3K27Ac. a. Jurkate E6 cells transfected with SRSF2 siRNAs or negative control siRNAs were cultured in poly-D-lysine-coated plates, fixed, incubated with an anti-STAT3 Y705p antibody (red), and anti-H3K27Ac antibody (green), and subjected to a confocal analysis. The intensity plots of the red and green channels were analyzed with ImageJ software. Scale bars, $10 \mu \mathrm{m}$. b. Jurkate E6 cell lysates transfected with SRSF2 siRNAs or negative control siRNAs were collected and immuno-precipitated with an anti-STAT3 Y705p antibody or anti-IgG antibody, followed by a western blot analysis of STAT3 Y705p, H3K27Ac, Histone $\mathrm{H} 3$, and $\beta$-actin. (C). Jurkate E6 cells were cultured in poly-

STAT3 to H3K27Ac by interrupting the association between STAT3 and H3K27Ac. Our data indicate that SRSF2 may function as an epigenetic regulator.

Taken together, this study reveals that most of TILs isolated from RCC tissue are exhausted and downregulation of SRSF2 in these TILs could significantly improve their immune response against tumor cells through inhibiting the expression of multiple immune checkpoint molecules via the alteration of
D-lysine-coated plates, fixed, incubated with an anti-SRSF2 antibody (red), anti-H3K27Ac antibody (green) or anti-STAT3 Y705p antibody (green), and subjected to a confocal analysis. The intensity plots of the red and green channels were analyzed with ImageJ software. Scale bars, $10 \mu \mathrm{m}$. d. Jurkate E6 cell lysates were collected and immuno-precipitated with an anti-SRSF2 antibody or anti-IgG antibody, followed by a western blot analysis of H3K27Ac and STAT3 Y705p. e. Schematic model of the roles of SRSF2 in P300/CBPmediated H3K27 acetylation (1) and STAT3 Y705p-mediated gene transcription (2)

the epigenetic status of these genes. All these data suggest that SRSF2 may be a therapeutic target to block tumor-associated suppressive pathways. 


\section{Materials and methods}

\section{Patients and samples}

In total, 13 patients with histologically confirmed RCC undergoing partial nephrectomy at the Department of Urology of the First Affiliated Hospital of Shenzhen University Hospital between October 2016 and September 2017 were enrolled in the study. The study was approved by the Ethics Committee of the First Affiliated Hospital of Shenzhen University. All patients signed a written consent form. Tumor specimens of at least $1 \mathrm{~cm}^{3}$ were obtained from primary RCC tumors. Blood samples were collected prior to surgery, and peripheral blood mononuclear cells (PBMCs) were isolated from the blood samples.

\section{Generation of TILs and tumor cells and cell culture}

The generation of TILs was performed according to Dudley's protocol [55]. In brief, a single tumor fragment was placed in individual wells of a 24-well tissue culture plate with $2 \mathrm{~mL}$ of complete medium (CM) plus $6000 \mathrm{IU}$ per $\mathrm{mL}$ of rhIL-2 (Chiron Corp., Emeryville, CA). The CM consisted of RPMI 1640 (Life Technologies, 21875091), 25 mmol/L HEPES pH 7.2 (Life Technologies, 15630080), $10 \mathrm{U} / \mathrm{ml}$ penicillin-streptomycin (Life Technologies, 15140163), and $2 \mathrm{mmol} / \mathrm{L}$-glutamine (Life Technologies, 25030081), supplemented with $10 \%$ human serum (SigmaAldrich, H4522). The plates were placed in a humidified $37{ }^{\circ} \mathrm{C}$ incubator with $5 \% \mathrm{CO}$. Half of the medium was changed at day 5 and thereafter every 2-3 days thereafter.

For generation of tumor cells, Single-cell suspensions were obtained from minced tumor by enzymatic digestion at $37{ }^{\circ} \mathrm{C}$ in $5 \mathrm{ml}$ of DMEM/F-12 (Life Technologies, 11320033 ) supplemented with $5 \mathrm{mg} / \mathrm{mL}$ collagenase type II (Life Technologies, 17101015), $30 \mathrm{U} / \mathrm{ml}$ DNAse I (Sigma-Aldrich, D5025), and $10 \mu \mathrm{M} \mathrm{Y-27632} \mathrm{dihydro-}$ chloride (AbMole, M1817). After $1 \mathrm{~h}$ for digestion, the tumor cell suspensions were centrifuged, resuspended, and filtered through $40 \mu \mathrm{m}$ cell strainer (Sigma-Aldrich, CLS431750-50EA) and then were cultured in DMEM/F12 supplemented with 20\% FBS (Gibco, 10270), 1\% Glutamax (Life Technologies, 35050038), 1\% HEPES, $5 \mathrm{ng} /$ mL FGF2 (Peprotech, 100-18B), 5 ng/mL EGF (Peprotech, AF-100-15), $5 \mathrm{mg} / \mathrm{L}$ insulin (Sigma-Aldrich, I9278), $5 \mathrm{mg} / \mathrm{L}$ transferrin (Sigma-Aldrich, T3309), $25 \mu \mathrm{g} / \mathrm{L}$ hydrocortisone (Sigma-Aldrich, H0888), and $10 \mu \mathrm{M}$ Y-27632 dihydrochloride (AbMole, M1817) in a humidified $5 \% \mathrm{CO} 2$ incubator at $37{ }^{\circ} \mathrm{C}$.

Jurkate E6 cells (American Type Culture Collection, ATCC) were grown in RPMI 1640 media supplemented with L-glutamine, $10 \%$ fetal bovine serum (PAA, A15101), and 1\% Penicillin/Streptomycin (Gibco/Invitrogen Ltd, 15140-122) in a humidified 5\% CO2 incubator at $37^{\circ} \mathrm{C}$.

\section{Data set}

A RCC data set (GSE40435) was downloaded from Gene Expression Omnibus (GEO, https://www.ncbi.nlm.nih.gov/ $\mathrm{gds} /$ ) database. The expression of SRSF2 and STAT3 in the RCC tumor tissue and adjacent tumor tissue was analyzed with nonparametric Kolmogorov-Smirnov test.

\section{Cell transfection, RNA isolation, reverse transcription, and qPCR}

All of the synthetic siRNAs and the negative control (siCTRL) siRNA were purchased from Shanghai GenePharma Co., Ltd. All the siRNAs were transfected with Neon ${ }^{\circledR}$ Transfection System (Invitrogen, MPK5000) according to the manufacturer's protocol. The sequences of the siRNAs used are listed in Table S1. Total RNA was isolated with RNAiso Plus (Takara, D9108B) according to the manufacturer's protocol. Real-time PCR was performed with ReverTra Ace ${ }^{\circledR}$ qPCR RT Master Mix with gDNA remover (Toyobo, FSQ-301) and SYBR Green PCR Master Mix (Toyobo, QPK-201). All mRNA levels were measured and normalized to that of $\beta$-actin mRNA. The primers used are listed in Table $\mathrm{S} 1$.

\section{Western blotting}

Cells were lysed in ice-cold whole-cell extract buffer B (50 mM Tris- $\mathrm{HCl}$ [pH 8.0], $4 \mathrm{M}$ urea, and 1\% Triton X-100) supplemented with complete protease inhibitor cocktail (Roche). The cell extracts were resolved with SDS-PAGE and analyzed with western blotting. The protein bands were visualized with ECL Blotting Detection Reagents. The antibodies used for western blotting included an antiSRSF2 antibody (Santa Cruz Biotechnology, sc-10252), anti-H3K27Ac antibody (Abcam, ab4729), anti-Histone H3 antibody (Abcam, ab1791), and anti- $\beta$-actin antibody (Proteintech, 60008-1-Ig).

\section{Flow cytometry}

To investigate the functions of SRSF2 in the immune response of TILs against autologous tumor cell lines, TILs transfected with SRSF2 siRNA were co-cultured with autologous tumor cell lines. Then, flow cytometry was performed with an anti-CD8 antibody (Biolegend, 344704) and anti-IFN- $\gamma$ antibody (BD Biosciences, 554552) on BD AccuriC6 (BD Biosciences). To examine the expression 
level of the immune checkpoints in the TILs and PBMCs, the frequency of immune checkpoint + and CD8 + TILs was assessed using flow cytometry on BD AccuriC6 (BD Biosciences). The antibodies against the immune checkpoints used for flow cytometry included PD-L1 (BD Biosciences, 561787), BTLA (BD Biosciences, 564802), CTLA-4 (BD Biosciences, 561717), LAG3 (BD Biosciences, 565617), and CD160 (BD Biosciences, 562351). All data were analyzed by FlowJo software.

\section{Luciferase assay}

For generation of luciferase reporters for promoter assay, three luciferase reporter constructs that inserted the sequences from $-500 \mathrm{bp}$ to $+500 \mathrm{bp}$ relative to the TSS of PD-L1, BTLA, CTLA-4, LAG3 and CD160 were purchased from Shanghai GenePharma Co. Ltd. Luciferase activities were assayed using a Dual-Luciferase Reporter System (Promega, E1960) according to the manufacturer's protocol.

\section{ChIP assay}

To investigate the roles of SRSF2 in the status of histone modification near the TSS of genes, ChIP assay was conducted according to Dahl's protocol [56]. In brief, $1 \times 10^{6}$ Jurkate E6 cells transfected with SRSF2 siRNA or control siRNAs were fixed with $1 \%$ formaldehyde and sonicated on ice for $10 \times 10 \mathrm{~s}$, with $20 \mathrm{~s}$ pauses between each $10 \mathrm{~s}$ session and $30 \%$ power using a probe sonicator (Sonics \& Materials, Inc.). After centrifugation, the supernatants were incubated with anti-H3K4Me3 antibody (Abcam, ab8580), anti-H3K27Me3 antibody (Abcam, ab6002), anti-H3K27 Ac antibody (Abcam, ab4729), or anti-STAT3 Y705 antibody (Abcam, ab76315). Chromatin DNA was purified by Dynabeads protein G (Invitrogen, 10004D) and subjected to realtime PCR. The region-specific primers used are listed in Table S1.

\section{Immunoprecipitation assay}

The immunoprecipitation assay was performed using the Immunoprecipitation Protein G Dynabeads ${ }^{\circledR}$ kit (Invitrogen, 10007D) according to the manufacturer's protocol. The antibodies used for immunoprecipitation included antiSRSF2 antibody (Abcam, ab11826), anti-P300 antibody (Abcam, ab59240), anti-CBP antibody (Abcam, ab50702), anti-STAT3Y705p antibody (Abcam, ab76315), and antiH3K27AC antibody (Abcam, ab4729).

\section{Immunofluorescence microscopy}

To determine the relationship between SRSF2 and P300/ CBP, the Jurkate E6 were cultured in Poly-D-lysine-coated plates, fixed with $4 \%$ paraformaldehyde, and incubated with anti-SRSF2 antibody (Abcam, ab11826), antiP300 antibody (Abcam, ab59240), or anti-CBP antibody (Abcam, ab50702) for $1.5 \mathrm{~h}$ at room temperature. After the cells were washed and incubated with the secondary antibody, they were counterstained with DAPI and mounted for observation. Cell images were obtained with an Olympus FV1000 confocal microscope. To study the role of SRSF2 in the interaction between STAT3 Y705p and H3K27Ac, the Jurkate E6 transfected with SRSF2 siRNA or negative control siRNA were cultured in Poly-D-lysine-coated plates, fixed with $4 \%$ paraformaldehyde, and incubated with the anti-STAT3 Y705 antibody (Abcam, ab76315) and anti-H3K27Ac antibody (Abcam, ab4729) for $1.5 \mathrm{~h}$ at room temperature. After the cells were washed and incubated with the secondary antibody, they were counterstained with DAPI and mounted for observation. Cell images were obtained with an Olympus FV1000 confocal microscope. To determine the relationship between SRSF2, STAT3 Y705p, and H3K27Ac, the Jurkate E6 were cultured in Poly-D-lysine-coated plates, fixed with 4\% paraformaldehyde, and incubated with anti-SRSF2 antibody (Abcam, ab11826), anti-STAT3 Y705 antibody (Abcam, ab76315), or anti-H3K27Ac antibody (Abcam, $\mathrm{ab} 4729)$ for $1.5 \mathrm{~h}$ at room temperature. After the cells were washed and incubated with the secondary antibody, they were counterstained with DAPI and mounted for observation. Cell images were obtained with an Olympus FV1000 confocal microscope.

\section{Statistics}

Data are expressed as mean \pm SD with repeated three times $(* p<0.001)$. Comparisons between two groups were evaluated using a two-sample $t$ test. For three or more groups, standard one-way analysis of variance (ANOVA) with Bonferroni's test was conducted. A two-tailed probability value $<0.05$ was considered to be statistically significant.

Acknowledgements This work was supported by the National Key Research and Development Program of China (2019YFA090015), National Natural Science Foundation of China (81772737), National Science Foundation Projects of Guangdong Province (2017B030301015), the Shenzhen Municipal Government of China (JCYJ20170413161749433 and JSGG20160301161836370), the Sanming Project of Shenzhen Health and Family Planning Commission (SZSM201412018, SZSM201512037), the high-level university's medical discipline construction (2016031638), and the Postdoctoral Research Foundation of China (2018M633216). 
Author contributions $\mathrm{ZW}, \mathrm{WH}$, and $\mathrm{ZC}$ designed the study and wrote the paper. ZW, KL, XW, YH, WW, WW, and WL finished experiments. $\mathrm{ZW}, \mathrm{WH}$, and ZC prepared all figures. All authors analyzed the results and approved the final version of the manuscript.

\section{Compliance with ethical standards}

Conflict of interest The authors have declared that no conflict of interest exists.

Open Access This article is distributed under the terms of the Creative Commons Attribution 4.0 International License (http://creativeco mmons.org/licenses/by/4.0/), which permits unrestricted use, distribution, and reproduction in any medium, provided you give appropriate credit to the original author(s) and the source, provide a link to the Creative Commons license, and indicate if changes were made.

\section{References}

1. Rini BI, Campbell SC, Escudier B (2009) Renal cell carcinoma. Lancet 373(9669):1119-1132

2. Jemal A, Thomas A, Murray T, Thun M (2002) Cancer statistics, 2002. CA Cancer J Clin 52(1):23-47

3. Schendel DJ et al (1997) Cellular and molecular analyses of major histocompatibility complex (MHC) restricted and nonMHC-restricted effector cells recognizing renal cell carcinomas: problems and perspectives for immunotherapy. J Mol Med (Berl) 75(6):400-413

4. Verra $\mathrm{N}$ et al (2005) Infiltration of activated dendritic cells and $\mathrm{T}$ cells in renal cell carcinoma following combined cytokine immunotherapy. Eur Urol 48(3):527-533

5. Bromwich EJ et al (2003) The relationship between T-lymphocyte infiltration, stage, tumour grade and survival in patients undergoing curative surgery for renal cell cancer. Br J Cancer 89(10):1906-1908

6. Nakano O et al (2001) Proliferative activity of intratumoral CD8(+) T-lymphocytes as a prognostic factor in human renal cell carcinoma: clinicopathologic demonstration of antitumor immunity. Cancer Res 61(13):5132-5136

7. Rabinovich GA, Gabrilovich D, Sotomayor EM (2007) Immunosuppressive strategies that are mediated by tumor cells. Annu Rev Immunol 25:267-296

8. Munn DH, Bronte V (2016) Immune suppressive mechanisms in the tumor microenvironment. Curr Opin Immunol 39:1-6

9. Gajewski TF, Schreiber H, Fu YX (2013) Innate and adaptive immune cells in the tumor microenvironment. Nat Immunol 14(10):1014-1022

10. Zou W (2005) Immunosuppressive networks in the tumour environment and their therapeutic relevance. Nat Rev Cancer 5(4):263-274

11. Miao D et al (2018) Genomic correlates of response to immune checkpoint therapies in clear cell renal cell carcinoma. Science 359(6377):801-806

12. Pan D et al (2018) A major chromatin regulator determines resistance of tumor cells to T cell-mediated killing. Science 359(6377):770-775

13. Cao K et al (2015) Histone deacetylase inhibitors prevent activation-induced cell death and promote anti-tumor immunity. Oncogene 34(49):5960-5970

14. Xiao R et al (2007) Splicing regulator SC35 is essential for genomic stability and cell proliferation during mammalian organogenesis. Mol Cell Biol 27(15):5393-5402
15. Lin $\mathrm{S}, \mathrm{Fu} \mathrm{XD}$ (2007) SR proteins and related factors in alternative splicing. Adv Exp Med Biol 623:107-122

16. Cartegni L, Chew SL, Krainer AR (2002) Listening to silence and understanding nonsense: exonic mutations that affect splicing. Nat Rev Genet 3(4):285-298

17. Tripathi $\mathrm{V}$ et al (2010) The nuclear-retained noncoding RNA MALAT1 regulates alternative splicing by modulating SR splicing factor phosphorylation. Mol Cell 39(6):925-938

18. Huang Y, Steitz JA (2005) SRprises along a messenger's journey. Mol Cell 17(5):613-615

19. Merdzhanova G, Edmond V, De Seranno S, Van den Broeck A, Corcos L, Brambilla C, Brambilla E, Gazzeri S, Eymin B (2008) E2F1 controls alternative splicing pattern of genes involved in apoptosis through upregulation of the splicing factor SC35. Cell Death Differ 15(12):1815-1823

20. Luo C, Cheng Y, Liu Y, Chen L, Liu L, Wei N, Xie Z, Wu W, Feng Y (2017) SRSF2 regulates alternative splicing to drive hepatocellular carcinoma development. Cancer Res 77(5):1168-1178

21. Kędzierska H, Popławski P, Hoser G, Rybicka B, Rodzik K, Sokół E, Bogusławska J, Tański Z, Fogtman A, Koblowska M, Piekiełko-Witkowska A (2016) Decreased expression of SRSF2 splicing factor inhibits apoptotic pathways in renal cancer. Int $\mathbf{J}$ Mol Sci. 17(10):1598

22. Moulton VR, Grammatikos AP, Fitzgerald LM, Tsokos GC (2013) Splicing factor SF2/ASF rescues IL-2 production in T cells from systemic lupus erythematosus patients by activating IL-2 transcription. Proc Natl Acad Sci U S A. 110(5):1845-1850

23. Makishima $\mathrm{H}$ et al (2012) Mutations in the spliceosome machinery, a novel and ubiquitous pathway in leukemogenesis. Blood 119(14):3203-3210

24. Maldarelli F, Xiang C, Chamoun G, Zeichner SL (1998) The expression of the essential nuclear splicing factor SC35 is altered by human immunodeficiency virus infection. Virus Res 53(1):39-51

25. Wang HY, Xu X, Ding JH, Bermingham JR Jr, Fu XD (2001) SC35 plays a role in T cell development and alternative splicing of CD45. Mol Cell 7(2):331-342

26. Loh TJ et al (2014) SC35 promotes splicing of the C5-V6-C6 isoform of CD44 pre-mRNA. Oncol Rep 31(1):273-279

27. Yi LX, Gao B, Gao F, Fu FQ, Zhang XG, Sun J (2012) Splicing factor SC35 regulated the expression of B7-H3 in vitro. Xi Bao Yu Fen Zi Mian Yi Xue Za Zhi 28(8):815-817

28. Wang $Z$ et al (2016) Serine/arginine-rich splicing factor 2 modulates herpes simplex virus type 1 replication via regulating viral gene transcriptional activity and pre-mRNA splicing. J Biol Chem 291(51):26377-26387

29. Janicki CN, Jenkinson SR, Williams NA, Morgan DJ (2008) Loss of CTL function among high-avidity tumor-specific CD8 + T cells following tumor infiltration. Cancer Res 68(8):2993-3000

30. Bendle GM et al (2004) Induction of unresponsiveness limits tumor protection by adoptively transferred MDM2-specific cytotoxic T lymphocytes. Cancer Res 64(21):8052-8056

31. Bai A, Higham E, Eisen HN, Wittrup KD, Chen J (2008) Rapid tolerization of virus-activated tumor-specific CD8 + T cells in prostate tumors of TRAMP mice. Proc Natl Acad Sci USA 105(35):13003-13008

32. Okoye IS, Houghton M, Tyrrell L, Barakat K, Elahi S (2017) Coinhibitory receptor expression and immune checkpoint blockade: maintaining a balance in $\mathrm{CD} 8+\mathrm{T}$ cell responses to chronic viral infections and cancer. Front Immunol 8:1215

33. Kimura H (2013) Histone modifications for human epigenome analysis. J Hum Genet 58(7):439-445

34. Thompson PR et al (2004) Regulation of the p300 HAT domain via a novel activation loop. Nat Struct Mol Biol 11(4):308-315

35. Kraus WL, Manning ET, Kadonaga JT (1999) Biochemical analysis of distinct activation functions in p300 that enhance 
transcription initiation with chromatin templates. Mol Cell Biol 19(12):8123-8135

36. Yu H, Pardoll D, Jove R (2009) STATs in cancer inflammation and immunity: a leading role for STAT3. Nat Rev Cancer 9(11):798-809

37. Wang Y et al (2016) AS-IL6 promotes glioma cell invasion by inducing H3K27Ac enrichment at the IL6 promoter and activating IL6 transcription. FEBS Lett 590(24):4586-4593

38. Goldstein I, Paakinaho V, Baek S, Sung MH, Hager GL (2017) Synergistic gene expression during the acute phase response is characterized by transcription factor assisted loading. Nat Commun 8(1):1849

39. Hossain DM et al (2014) Leukemia cell-targeted STAT3 silencing and TLR9 triggering generate systemic antitumor immunity. Blood 123(1):15-25

40. Hutchins AP, Poulain S, Miranda-Saavedra D (2012) Genomewide analysis of STAT3 binding in vivo predicts effectors of the anti-inflammatory response in macrophages. Blood 119(13):e110-119

41. Dou Z, Berger SL (2018) Senescence elicits stemness: a surprising mechanism for cancer relapse. Cell Metab 27(4):710-711

42. Milanovic $\mathrm{M}$ et al (2018) Senescence-associated reprogramming promotes cancer stemness. Nature 553(7686):96-100

43. Shi K, Haynes M, Huang L (2017) Nanovaccines for remodeling the suppressive tumor microenvironment: new horizons in cancer immunotherapy. Front Chem Sci Eng 11(4):676-684

44. Sharma P, Allison JP (2015) The future of immune checkpoint therapy. Science 348(6230):56-61

45. Herbst RS et al (2016) Pembrolizumab versus docetaxel for previously treated, PD-L1-positive, advanced non-small-cell lung cancer (KEYNOTE-010): a randomised controlled trial. Lancet 387(10027): $1540-1550$

46. Borghaei $\mathrm{H}$ et al (2015) Nivolumab versus docetaxel in advanced nonsquamous non-small-cell lung cancer. N Engl J Med 373(17):1627-1639

47. Brahmer J et al (2015) Nivolumab versus docetaxel in advanced squamous-cell non-small-cell lung cancer. N Engl J Med 373(2):123-135
48. Powles $\mathrm{T}$ et al (2014) MPDL3280A (anti-PD-L1) treatment leads to clinical activity in metastatic bladder cancer. Nature 515(7528):558-562

49. Fehrenbacher L et al (2016) Atezolizumab versus docetaxel for patients with previously treated non-small-cell lung cancer (POPLAR): a multicentre, open-label, phase 2 randomised controlled trial. Lancet 387(10030):1837-1846

50. Rosenberg JE et al (2016) Atezolizumab in patients with locally advanced and metastatic urothelial carcinoma who have progressed following treatment with platinum-based chemotherapy: a single-arm, multicentre, phase 2 trial. Lancet 387(10031):1909-1920

51. Cousin S, Italiano A (2016) Molecular pathways: immune checkpoint antibodies and their toxicities. Clin Cancer Res 22(18):4550-4555

52. Johnson DB et al (2016) Ipilimumab therapy in patients with advanced melanoma and preexisting autoimmune disorders. JAMA Oncol 2(2):234-240

53. Sen DR et al (2016) The epigenetic landscape of T cell exhaustion. Science 354(6316):1165-1169

54. Pauken KE et al (2016) Epigenetic stability of exhausted T cells limits durability of reinvigoration by PD-1 blockade. Science 354(6316):1160-1165

55. Dudley ME, Wunderlich JR, Shelton TE, Even J, Rosenberg SA (2003) Generation of tumor-infiltrating lymphocyte cultures for use in adoptive transfer therapy for melanoma patients. J Immunother 26(4):332-342

56. Dahl JA, Collas P (2008) A rapid micro chromatin immunoprecipitation assay (microChIP). Nat Protoc 3(6):1032-1045

Publisher's Note Springer Nature remains neutral with regard to jurisdictional claims in published maps and institutional affiliations. 